\title{
Semi-analytical Method for Predicting Shaft Voltage in Field-excited Synchronous Generators
}

\author{
Wesley Doorsamy ${ }^{\dagger}$ and Willem A. Cronje* \\ $\dot{\dagger}^{*}$ Dept. of Electrical and Information Engineering, University of the Witwatersrand, Johannesburg, South Africa
}

\begin{abstract}
This study presents an electromagnetic model for predicting shaft voltages in a 2-pole field-excited synchronous generator. After the first observations on shaft voltages were made more than a century ago, extensive work has been conducted on eliminating, mitigating, and integrating the aforementioned phenomena. Given that emphasis has been placed on modeling shaft- and bearing-induced voltages in AC motors driven by variable frequency drives, similar efforts toward a model that is dedicated to generators are insubstantial. This work endeavors to improve current physical interpretation and prediction methods for shaft-induced voltages in generators through semi-analytical derivation. Aside from the experimental validation of the model, investigations regarding the behavior of shaft voltages under varying machine complexities and operating conditions clarify previous uncertainties regarding these phenomena. The performance of the numerical method is also assessed for application in eccentricity fault diagnosis.
\end{abstract}

Key words: 2-pole synchronous generator, Fault diagnosis, Shaft voltage model

\section{INTRODUCTION}

The phenomena of shaft voltages in electrical rotating machines were initially observed more than a century ago. The scope of research and development in this area has subsequently ranged from protection strategies (shaft voltage mitigation) in generators and machines with variable frequency drives (VFDs) to condition monitoring applications in generators. Although many interpretations of shaft voltages have been offered during this period, no clear theoretical description is available that accounts for the numerous characteristics of these phenomena. These theoretical shortcomings are particularly evident in the case of synchronous machines, wherein the origins and influential parameters of shaft voltages are uncertain.

Shaft voltages in generators are significant from both protection and condition monitoring perspectives [1], [2]. Previous works have indicated that for protection purposes, predicting the induced voltage levels as early as the design stage is useful. With condition monitoring and preventive maintenance, in particular, accurately relating minor variations

Manuscript received Apr. 17, 2014; accepted Jul. 14, 2014 Recommended for publication by Associate Editor Tae-Won Chun.

†Corresponding Author: wesley.doorsamy@students.wits.ac.za Tel: +27-11-717-7204, Fax: +27-11-403-1929, Univ. of the Wits.

*Department of Electrical and Information Engineering, University of the Witwatersrand, South Africa in shaft voltages to specific machine parameters is advantageous.

However, an adequate theoretical framework for modeling shaft voltages has not yet been proposed. This study extends an initial work [3] that aimed to address some of the aforementioned shortcomings, particularly with regard to synchronous generators. Such generators are widely used in power stations worldwide. The conventional 2-pole machine uses a field winding with static DC excitation and a solid cylindrical rotor.

A method for accurately predicting shaft voltages in synchronous generators is derived from fundamental electromagnetic theory by using a semi-analytical approach. Substantial augmentation of the initial work includes a detailed deliberation of the construction and implementation of the finite element (FE) model. A systematic validation is also provided through theoretical substantiation and accuracy estimation via an experimental system. The proposed shaft voltage model is also tested in the context of fault diagnosis application.

\section{BACKGROUND}

Shaft voltages were initially discovered as a major root cause of bearing failure (one example of the earliest reports was published by a manufacturer in [4]). This scenario 
prompted extensive research and development efforts to mitigate the aforementioned effects as early as 1910 [5].

Efforts to control and eliminate the problems caused by shaft voltages have endured with notable works such as [6], [7]. Recognizing shaft voltages in turbine generators was illustrated in the early 1970s through the publication of guidelines that initiate an increasingly standard utilization of techniques to mitigate shaft voltages such as insulated bearings and grounding brushes [8]. The behavior of shaft voltages during this period was presented along with different models to characterize the observed phenomena. Examples of these models, which utilize capacitive coupling and other effects such as non-ideal DC field excitation or three-phase rectifiers, are provided in [9] and [10]. Many circuit models that characterize shaft voltages and currents have also been proposed, such as the model presented in [11].

Many of these earlier models do not account for several characteristics of shaft voltages such as different constituent harmonics. Furthermore, physical interpretation and the exact origins of these induced voltages depend nearly completely on experimental data.

The presented theoretical framework and predictive model for shaft voltages in 2-pole synchronous generators attempts to resolve some of the aforementioned issues by providing both quantitative and qualitative information.

\section{Construction of A Shaft Voltage Model}

\section{A. Measurement Concept}

A semi-analytical approach is used to model shaft voltages by applying fundamental electromagnetic theory to derive a theoretical model from a conceptual model. A physical interpretation or conceptualization of shaft voltages in synchronous generators is thus a prerequisite for the presented analytical methodology. The measurement concept for the phenomena is considered to obtain a physical interpretation of shaft voltages (Fig. 1). A shaft voltage is measured as the potential across "a" and "d," where point "a" is typically the outboard or static excitation end and point " $\mathrm{d}$ " is the inboard or driven end.

When the shaft is under the action of a prime mover and the rotor windings are supplied with DC excitation, a rotating magnetomotive force (MMF) that is spatially sinusoidal under ideal conditions is generated. The net flux (through the measurement loop shown in Fig. 1) remains constant over time for a constant shaft speed under ideal conditions.

High-frequency components arise because of the slots on the stator and rotor core in a practical machine. The net flux through the loop also fluctuates with varying magnetic fields (rotating excitation fields) under non-ideal conditions, such as non-ideal DC source, field, and machine asymmetries.

This condition results in a time-varying net flux through loop "abcd" (surface on loop plane), and thus, induces a

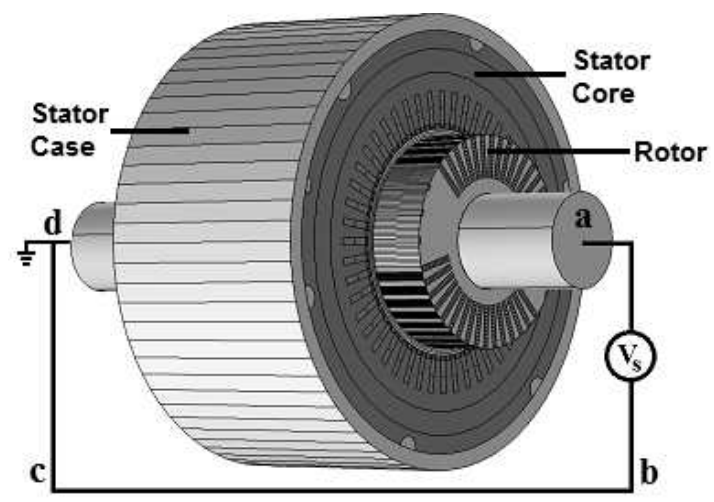

Fig. 1. Illustration of shaft voltage measurement on a synchronous generator (that is, across length "ad," where point "d" is grounded).

voltage across the shaft.

An example of a variation in the net flux over time occurs in the case of a non-uniform air gap. A traveling MMF wave is subjected to varying air-gap permeances and results in an asymmetrical flux. The time-varying net flux through the area of the loop induces a current in the loop, and thus, a voltage is induced across "ad." The return path "bc" consists of a shaft voltage sensor that measures the voltage across "a" (excitation end) and "d" (grounded driven end) in the case of experimental measurement. The presented concept for shaft voltages can be summarized analytically to simply imply the following:

$$
\mathrm{V}_{\mathrm{S}}>0 \text { when } \Delta \phi_{a c b d}>0 \text { for } \omega, \mathrm{I}_{\mathrm{FIELD}}=\text { constant } .
$$

This concept is used to construct and implement a predictive model by employing FE methods.

\section{B. FE Model}

An FE model of the experimental generator (Fig. 2) is constructed for this investigation. The model is designed for both transient and steady-state magnetic applications and enables coupling with an external electric circuit. The induced currents, along with the skin and proximity effects in conducting regions, can be accommodated as necessary. Only the steady-state solution is considered for the purpose of the present investigation.

The solution to the magnetic problem is obtained by using Maxwell's equations combined with the constitutive formulas for the matter. The governing electromagnetic field equations used for the model (where state variables are time dependent) are listed as follows [12]:

$$
\begin{gathered}
\nabla \times \vec{E}=-\frac{\partial \vec{B}}{\partial t}, \\
\vec{B}=0, \\
\nabla \times \vec{H}=\vec{J},
\end{gathered}
$$




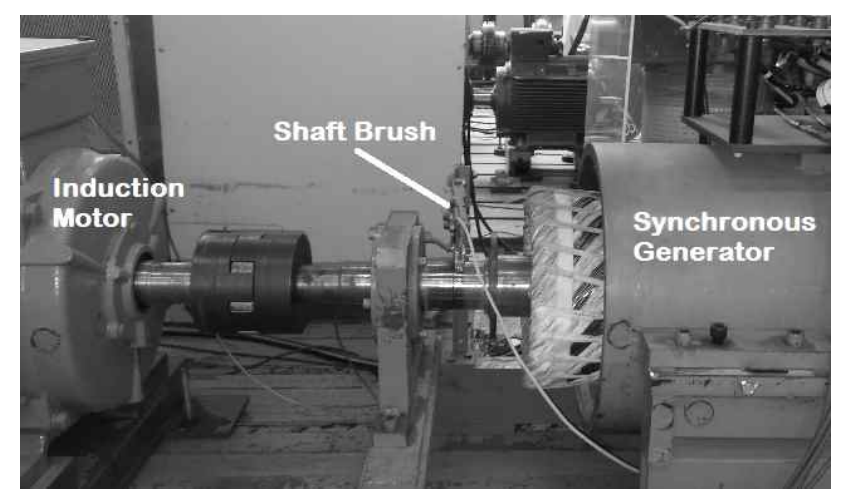

Fig. 2. Experimental setup with an induction motor (prime mover), shaft brush, and synchronous generator.

where $E$ is the electric field intensity $(\mathrm{V} / \mathrm{m}), B$ is the magnetic flux density $(\mathrm{T}), H$ is the magnetic field intensity $(\mathrm{A} / \mathrm{m})$, and $J$ is the current density $\left(\mathrm{A} / \mathrm{m}^{2}\right)$. The constitutive laws are also given by Equations (5) and (6) as follows:

$$
\begin{aligned}
& \vec{J}=\sigma \vec{E}, \\
& \vec{B}=\mu \vec{H},
\end{aligned}
$$

where $\sigma$ and $\mu$ are the electrical conductivity $\left(\Omega^{-1} \mathrm{~m}^{-1}\right)$ and the magnetic permeability $(\mathrm{H} / \mathrm{m})$ of the material, respectively.

The given Maxwell-Faraday relation, which can be represented by Equation (7), implies the presence of an electric scalar potential $V$ and a magnetic vector potential $A$.

$$
\vec{E}=\frac{\partial}{\partial t} \vec{A}-\nabla V
$$

A 2D plane model is suitable for the purpose of this investigation. However, certain assumptions for the 2D FE are intrinsic, such as magnetic field direction, current flow, and equal length of the stator and rotor cores.

Fig. 3 shows the modeled 2D geometry, which illustrates the flux path at rotor position $\theta=0^{\circ}$ of the generator used in this investigation. The geometric parameters are listed in Table II in the Appendix.

The magnetic flux travels through the rotor, air gap, and stator. The first step in obtaining the time-varying net flux is to consider path "AB," which corresponds to path "ab," shown in Fig. 1. The 2D case assumes that the machine is axially uniform, which inherently implies that the surface created by the loop is uniform along the length of the machine for an instant.

This condition allows the calculation of the net flux across a surface "ABCD" as an approximation of the actual loop "abcd" by considering the flux density over path "AB" and applying this throughout the length of the machine, that is, axial symmetry. Considering path "AB," the net instantaneous flux $(\mathrm{Wb} / \mathrm{m})$ may be computed by integrating the flux density (normal to the path) from points " $A$ " to "B," as shown in Equation (8). Fig. 4 shows the flux density along path "AB."

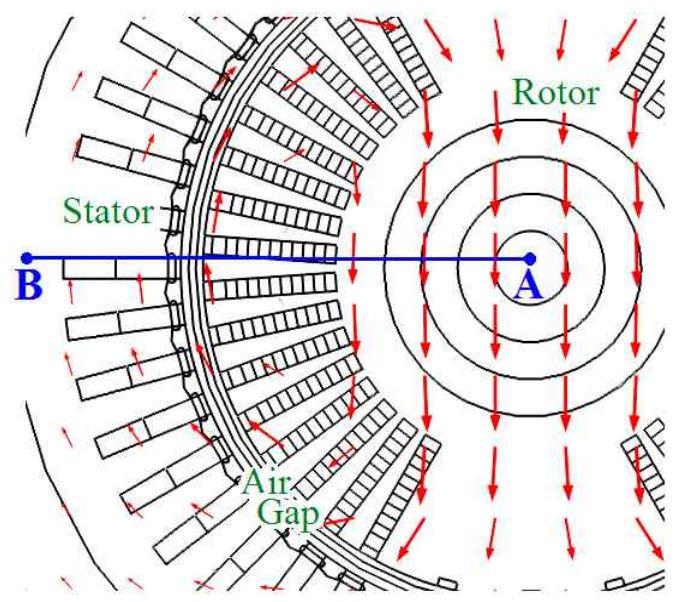

Fig. 3. Geometry and cross-sectional front view of the 2D FE model of a synchronous generator that illustrates path length "AB".

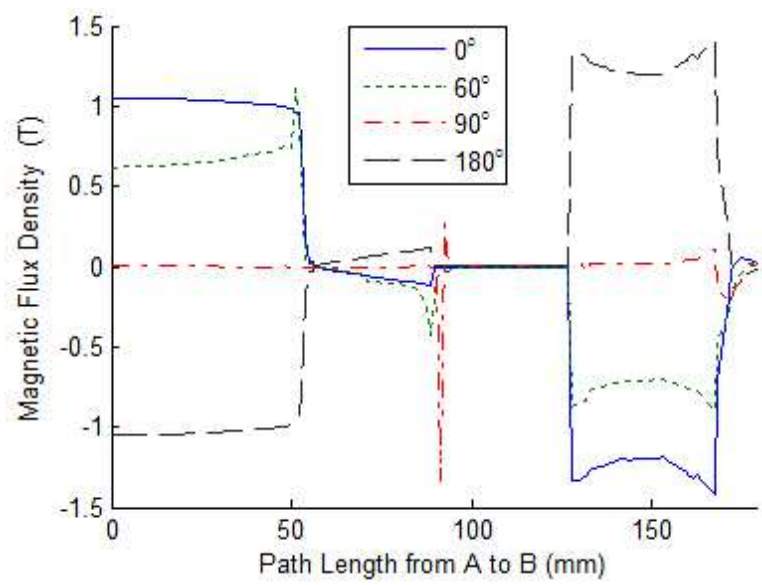

Fig. 4. Numerical solution to magnetic flux densities at different rotor positions over path "A" to "B" (Fig. 3).

$$
\phi_{A B}=\int_{A}^{B} \vec{B}_{\perp} d x
$$

The magnetic flux densities shown in Fig. 4 are recorded under normal conditions at $3000 \mathrm{rpm}$ with an excitation current of $40 \mathrm{~A}$. The integral given in Equation (8) is simply multiplied by the length $(L)$ of the machine given in Equation (9) to obtain the actual net flux $(\mathrm{Wb})$ through the surface corresponding to the 2D path "AB." Fig. 5 illustrates the complete process for shaft voltage computation.

$$
\phi_{A B C D}=L \int_{A}^{B} B_{\perp} d x
$$

The net flux integral is expected to be constant over time for an ideal machine. However, the net flux given by this integral does not remain constant over time for a practical machine with a slotted stator and rotor.

The instantaneous net flux is calculated at $1 \mathrm{~ms}$ intervals with a rotor displacement of approximately $18^{\circ}$ over a certain period by using the process shown in Fig. 5 . 


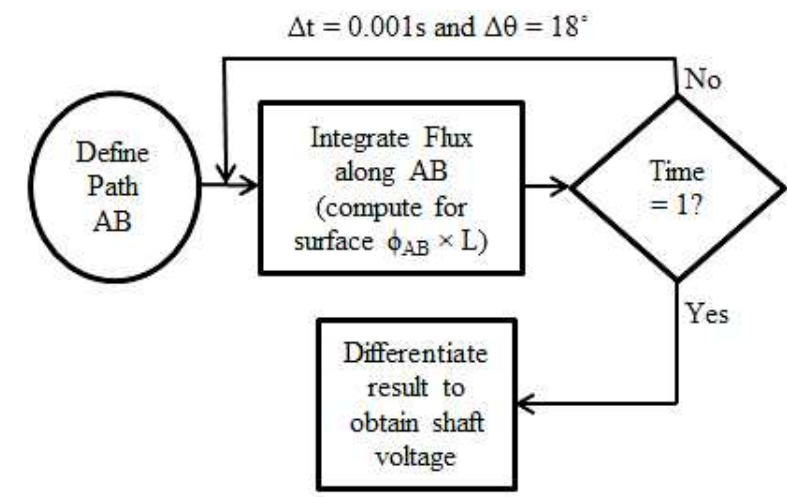

Fig. 5. Process flow diagram that describes the methodology used to obtain the numerical solution of the shaft voltage.

This condition allows for a steady-state stepped computation of the instantaneous net flux. The total flux is computed by iterating this process several times (for example, a waveform of a $1 \mathrm{~s}$ interval length will require 1000 iterations).

Equation (1) describes that the change in this resultant flux over the observation period is essentially the expected shaft voltage. The voltage induced within the loop "ABCD" is obtained by simply computing the flux linkage and its time derivative as provided by Faraday's law in Equations (10) and (11) as follows:

$$
\begin{aligned}
& \Phi_{A B C D}=N \phi_{A B C D}, \\
& V_{S}=-\frac{d}{d t} \Phi_{A B C D} .
\end{aligned}
$$

$N$ is defined as the relevant number of turns and indicates unity in the case of a solid shaft in the expression given in Equation (11).

The net instantaneous flux and shaft voltage waveforms obtained by using the presented numerical method under normal operating conditions without a load are shown in Fig. 6.

\section{TESTING AND APPLICATION}

\section{A. Theoretical Substantiation}

The validity of the presented model is first investigated by verifying if model behavior complies with theoretical conceptualization. The primary assumption that the shaft voltage is approximately zero under ideal conditions must be investigated. This assumption implies that the instantaneous net flux through the stipulated surface should be approximately zero, and thus, remains constant over time. This assumption is verified through examination of the net flux behavior over path "AB" (Fig. 3) by eliminating individual non-ideal components of the model.

The shaft voltage is obtained by using the presented methodology for different scenarios to diminish the complexity of the machine and move toward an ideal machine scenario (that is, no voltage is induced on the shaft).

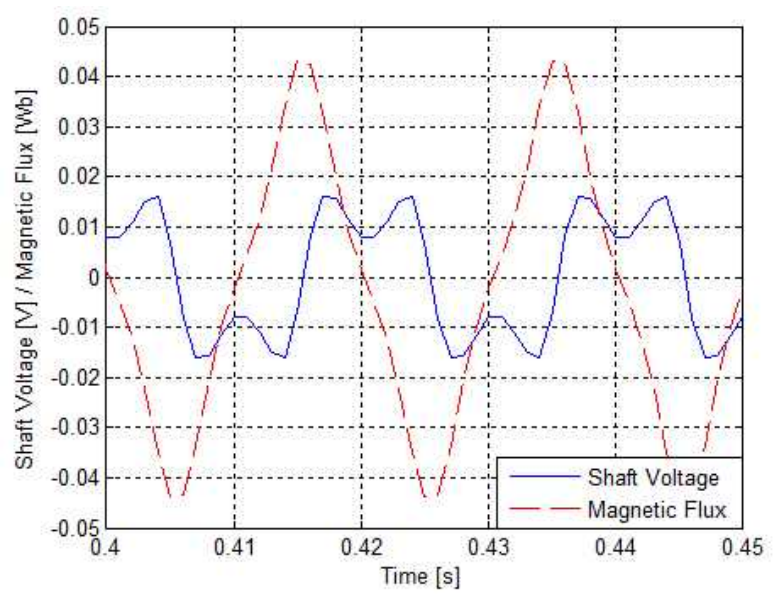

Fig. 6. Shaft voltage and corresponding flux waveforms under normal no-load conditions obtained by using the predictive model.

TABLE I

Shaft Voltage Amplitude for Diminished Machine COMPLEXITY AND MOVEMENT TOWARD IDEAL CONDITIONS

\begin{tabular}{lc}
\hline \multicolumn{1}{c}{ Scenario } & $\begin{array}{c}\text { Shaft voltage } \\
\text { amplitude }(\mathrm{mV})\end{array}$ \\
\hline All features with static eccentricity & 230 \\
All features of practical machine & 160 \\
No key way & 130 \\
Key ways and stator slots removed & 80 \\
$\begin{array}{l}\text { Key ways, stator slots, and rotor slots } \\
\text { removed }\end{array}$ & 30 \\
\hline
\end{tabular}

Table I shows the results of this investigation. The first scenario models a practical machine with a non-uniform air gap (that is, static eccentricity with a positive vertical displacement of $5 \%$ with respect to the air gap). Each scenario then removes a significant feature of the machine, such as stator case key ways which introduce machine asymmetries. The final scenario has no slot on the rotor and stator. A linear permanent magnet rotor with unidirectional magnetization is used to generate an ideal rotational field and provide excitation. The results indicate that a decreasing shaft voltage is removed as a practical feature of the machine as expected in the presented model. The effects of diminishing practical features are also not confined to a simple increase in the shaft voltage amplitude but also affect the frequency characteristics of the signal.

The shaft voltage recorded for the last scenario exhibits substantially lower levels and significantly reduced higher frequency harmonics than the practical machine scenario.

The results of a detailed analysis of the harmonics for each scenario show a definite trend for the 3rd, 5th, and 7th harmonics.

These high-frequency harmonics tend to decrease in 




Fig. 7. Experimental and modeled shaft voltage waveforms obtained under normal no-load conditions.

magnitude as practical features of the machine are removed (that is, from the first to the final scenario). Static eccentricity exhibits a $19 \%$ increase in the 6 th harmonic relative to the practical machine scenario.

Removing most practical machine features (that is, no key way as well as rotor and stator slot) results in decreases of $56 \%, 81 \%$, and $79 \%$ (relative to the second scenario or practical machine scenario) in the $3 \mathrm{rd}, 5 \mathrm{th}$, and 7 th harmonics, respectively. These results are significant to resolve some of the physical interpretation uncertainties with regard to variations in harmonics. The investigation also confirms that the model complies with the theoretical proposition for the origins of shaft voltages in synchronous generators.

\section{B. Experimental Validation}

Predicting the expected shaft voltages levels is advantageous for protection purposes. However, information regarding the harmonic content of the phenomena is essential with frequent use of shaft voltages for machine health diagnostics. This condition requires good accuracy for the modeled shaft voltage compared with experimental measurements. Thus, an analysis of the numerical results is conducted under laboratory conditions. An experimental miniature generator (Fig. 2) that was designed to mimic large turbo generators is used. The generator is a synchronous 2-pole machine rated at $20 \mathrm{kVA}$ and $3000 \mathrm{rpm}$ for an operational frequency of $50 \mathrm{~Hz}$. The generator is fitted with a shaft voltage monitoring system with the necessary preconditioning systems to eliminate noise and capture signals. The experimental and simulated shaft voltages are shown in Fig. 7. These waveforms are obtained under normal operating conditions $(50 \mathrm{~Hz}$ at $3000 \mathrm{rpm}$ ) without load. Each signal is normalized by using the following relation for comparison:

$$
V_{S}^{\prime}=\frac{\mathrm{V}_{\mathrm{S}}-\min \left(\mathrm{V}_{\mathrm{S}}\right)}{\max \left(\mathrm{V}_{\mathrm{S}}\right)-\min \left(\mathrm{V}_{\mathrm{S}}\right)}
$$



Fig. 8. Frequency content of the experimental and modeled shaft voltage signals under normal no-load conditions.

The actual instrumentation used to monitor and capture shaft voltages consists of a data acquisition system that is linked to the sensors through signal preconditioning systems. Specialized silver-gold alloy brushes are placed on the finished landings on either side of the rotor shaft.

The output of the brush device is fitted with anti-aliasing filters and connected to the analogue-to-digital inputs of the data acquisition system. The signal is a periodic voltage with a fundamental frequency that corresponds to the expected machine speed. The peaks represent the rotor poles, and the ripples are caused by the rotor teeth/slots. The error (that is, the difference between the model and the experimental results) is estimated to validate the accuracy of the FE model. Total model error $\left(E_{T}\right)$ for a particular number of samples $(N)$ is given by the following:

$$
E_{T}=\left\|\left(X_{S}-X_{E}\right)\right\|^{2},
$$

where $X_{S}$ is the simulated and $X_{E}$ is the measurement vectors. The average percentage error $\left(E_{A V E}\right)$ of the model may be calculated as follows by using the result from Equation (13):

$$
E_{A V E}=\left[\frac{E_{T} / N}{\max \left(V_{S}\right)-\min \left(V_{S}\right)}\right] \times 100 \% .
$$

This equation yields a value of $15.95 \%$. This value may be classified as percentage uncertainty. The sources of this error are not precisely known and are predominantly attributed to the unquantifiable physical characteristics of the machine and the measurement loop. However, the modeled signal content is mostly similar to that of the experimental content with regard to synchronism and predominant odd harmonics.

Some minor differences are observed when comparing the harmonic content of the modeled and experimental signals (Fig. 8). These differences are attributed to the following reasons. 
- Manufacturing tolerances of the experimental machine, such as core imperfections and rotor alignment, introduce relatively high nominal values particularly for the 5th and 6th harmonics.

- The experimental 3rd harmonic is significantly high relative to its fundamental counterpart. This condition is mainly attributed to the peak-to-peak ripple on the rectified excitation source.

- Another factor is the non-linearity of the magnetic material that is more evident at higher currents than at lower currents because of saturation.

- The experimental 3rd harmonic is relatively higher than the modeled signal because the model does not account for non-ideal DC excitation. The 3rd harmonic of the shaft voltage is attributed to the ripple produced by the three-phase rectifier; moreover, it is inherently contained in the flux density wave of a practical machine [13].

- Rotor end windings and a mush-wound stator can also contribute to differences in the modeled shaft voltage because these features are not part of the FE model.

\section{Application in Fault Diagnosis}

Further testing of the predictive model is conducted in the context of an application. Model performance in this case is assessed for application in incipient fault diagnosis (that is, condition monitoring on a synchronous generator). The previously described experimental generator is also designed to accommodate eccentricity faults under controlled conditions. The stator is specifically designed to be repositioned relative to the rotor, which is placed on pedestal bearings, and thus, allows implementation of static eccentricity. The machine stator is raised and $0.5 \mathrm{~mm}$-thick spacers are used to fix the stator at the modified position to obtain a precise level. The waveforms and corresponding FFTs obtained for the shaft voltage from the experimental measurements and the model under no-load condition with an eccentricity fault of $0.5 \mathrm{~mm}$ are shown in Figs. 9 and 10.

Some minor discrepancies are found mainly in the harmonic content, which is similar to the previous comparison of the normal operating model and the experimental signals. However, a substantially increased 6th harmonic is evident in both the modeled and experimental signals. The 6th harmonic $(300 \mathrm{~Hz})$ is the frequency used to diagnose eccentricity faults with shaft voltages. This condition demonstrates the accuracy with which the model can predict the behavior of the shaft voltage under fault conditions. The average percentage error $\left(E_{A V E}\right)$ for the case of the aforementioned fault is calculated as $10.49 \%$ through Equations (13) and (14). Model performance is improved under fault conditions because the experimental generator has a default eccentricity level resulting from manufacturing and commissioning tolerances.

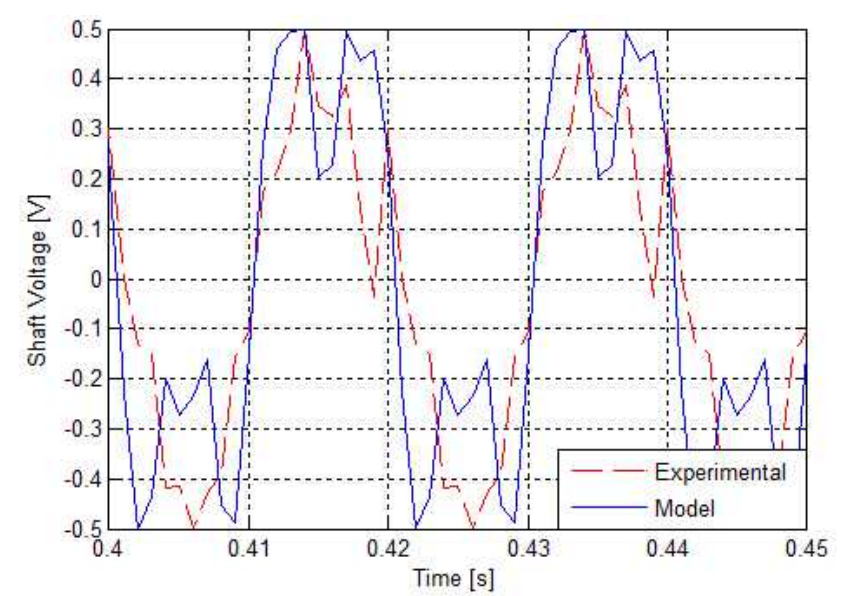

Fig. 9. Experimental and modeled shaft voltage waveforms under no-load conditions with $0.5 \mathrm{~mm}$ static eccentricity fault.

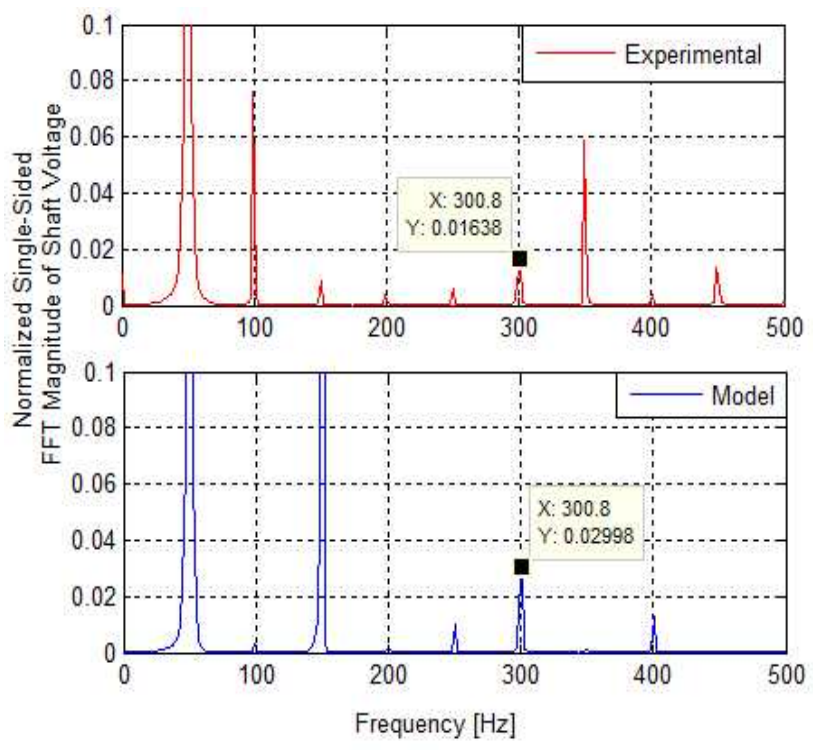

Fig. 10. Frequency content of the experimental and modeled shaft voltage signals under static eccentricity fault condition.

\section{RECOMMENDATIONS}

The presented testing of the predictive model agrees well with the proposed theoretical framework for shaft voltages in synchronous generators. However, minor differences between the predicted and experimental waveforms are observed as described in the experimental validation. These numerical differences are attributed to specific insufficiencies inherent to the 2D FE model. A 3D model, although potentially expensive, can resolve some of these differences by considering the end effects and most manufacturing tolerances of the machine. A detailed uncertainty analysis of shaft voltages can also eliminate differences between the model and experimental harmonics. In particular, a sensitivity analysis of the harmonics can qualitatively contribute to the refinement of the predictive model. 


\section{CONCLUSIONS}

A predictive model for shaft voltages in a synchronous generator is presented. An extensive investigation into previous models indicates that a suitable physical interpretation of this phenomenon is required. The availability of an accurate shaft voltage model is significant from both protection and condition monitoring perspectives.

The model is derived by applying a semi-analytical approach and the shaft voltage is identified as a rate of change of the net flux through the specified loop and surface. Construction and implementation of the model is carried out by using $2 \mathrm{D}$ FE. An analysis of the model under varying degrees of machine complexity validates the theoretical model and physical interpretation of shaft-induced voltages. Testing the numerical solution for the shaft voltage under normal operating conditions indicates good correlation with measurements obtained from an experimental generator.

The model also achieves high accuracy in predicting an incipient eccentricity fault. This condition demonstrates the suitability of the presented method for application in condition monitoring in synchronous generators. The model is thus considered as valuable in describing previously uncertain characteristics and accurately predicting shaft voltages for various applications.

\section{APPENDIX}

TABLE II

GeOMETRIC PARAMETERS OF THE EXPERIMENTAL SyNCHRONOUS GENERATOR

\begin{tabular}{lc}
\hline \multicolumn{1}{c}{ Parameter } & Value \\
\hline Number of stator slots & 48 \\
Number of rotor slots & 32 \\
Number of damper bars & 38 \\
Stator core & $355.6 \mathrm{~mm}(\mathrm{id}), 190.5 \mathrm{~mm}(\mathrm{od})$ \\
Rotor diameter & $178.5 \mathrm{~mm}$ \\
Stator slot & $5.8 \mathrm{~mm}(\mathrm{w}), 15 \mathrm{~mm}(\mathrm{~h})$ \\
Rotor slot & $5 \mathrm{~mm}(\mathrm{w}), 36 \mathrm{~mm}(\mathrm{~h})$ \\
Damper slot & $2.2 \mathrm{~mm}(\mathrm{w}), 5 \mathrm{~mm}(\mathrm{~h})$ \\
Core length & $280 \mathrm{~mm}$ \\
Air gap & $6 \mathrm{~mm}$ \\
\hline
\end{tabular}

\section{REFERENCES}

[1] P. I. Nippes, "Early warning of developing problems in rotating machinery as provided by monitoring shaft voltages and grounding currents," IEEE Trans. Energy Convers., Vol. 19, No. 2, pp. 340-345, Jun. 2004.

[2] J. Torlay, C. Corenwinder, A. Audoli, J. Herigault, and A. Foggia, "Analysis of shaft voltages in large synchronous generators," IEEE International Conference on Electrical
Machines and Drives, pp. 607-609, 1999.

[3] W. Doorsamy, and W. A. Cronje, "An electromagnetic model for shaft voltages in synchronous generators," in Proc. 15th Int. Conf. IEEE ICIT, pp. 149-154, 2014.

[4] F. Punga and W. Hess, "Bearing currents," Ekbrotechnik and Maschinenbau, Vol. 25, pp. 615-18, August 1907.

[5] L. Berger, "Causes of bearing currents and their elimination," La Lumiere Electrique, Vol. 11, pp. 268-17, Aug. 1910.

[6] P. L. Alger and H. W. Samson, "Shaft currents in electric machines," Transactions AIEE, pp. 235-245, 1945.

[7] J. Boyd and H. N. Kaufman, "The causes and control of electrical currents in bearings," Lubrication Engineering, pp. 28-35, Jan. 1959.

[8] ANSI/IEEE C50.30, "IEEE guide for operation and maintenance of turbine generators," IEEE, 1972.

[9] Y. C. Ammann, K. Reichert, R. Joho and Z. Posedel, "Shaft voltages in generators with static excitation systems problems and solutions," IEEE Trans. Energy Convers., Vol. 3, No. 2, pp. 409-419, Jun. 1988.

[10] I. Kerszenbaum, "Shaft currents in electric machines fed by solid-state drives", IEEE Proceedings of Annual Industrial and Commercial Power Systems Technical Conference, pp. 71-79, 1992.

[11] M. J. Costello, "Shaft voltages and rotating machinery," IEEE Trans. Ind. Appl., Vol. 29, No. 2, pp. 419-426, Mar./Apr. 1993.

[12] J. P. A. Bastos and N. Sadowski, Electromagnetic Modeling by Finite Element Methods, Marcel Dekker Inc., 2003.

[13] L. W. Matsch and J. D. Morgan, Electromagnetic and Electromechanical Machines, 3rd ed., John Wiley \& Sons, Inc.: Canada, pp. 176-186, 1986.

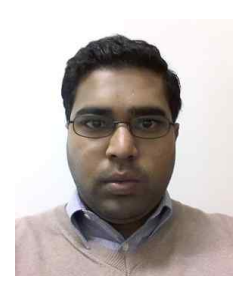

Wesley Doorsamy received his B.Sc. and M.Sc. in Electrical Engineering from the University of the Witwatersrand, Johannesburg, South Africa. He is currently a Ph.D. candidate at the said university with research interests in electrical machines.

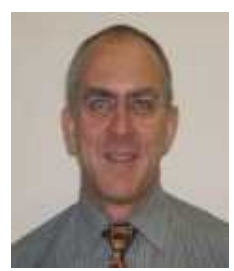

Willem A. Cronje obtained his D.Ing. from the Rand Afrikaans University in Johannesburg, South Africa. He works in power electronics and magnetic design, has co-authored several papers in the field, and currently holds a chair in Electrical Machines and Drives at the University of the Witwatersrand. 